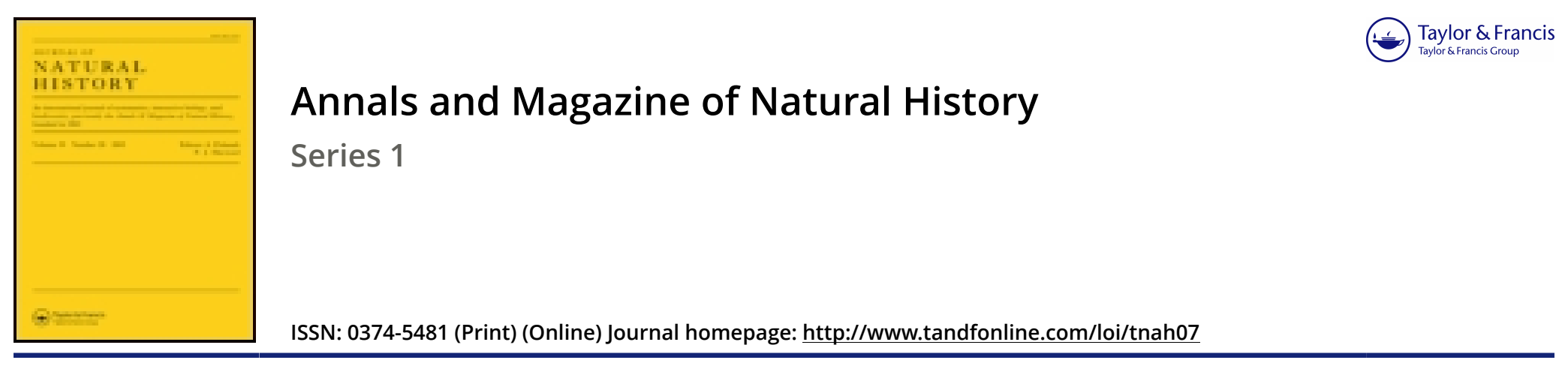

\title{
XXXIX.-Descriptions of Chalcidites discovered in Valdivia by C. Darwin., Esq.
}

\section{Francis Walker F.L.S.}

To cite this article: Francis Walker F.L.S. (1842) XXXIX.-Descriptions of Chalcidites discovered in Valdivia by C. Darwin., Esq., Annals and Magazine of Natural History, 10:65, 271-274, DOI: 10.1080/03745484209445234

To link to this article: http://dx.doi.org/10.1080/03745484209445234

册 Published online: 04 Dec 2009.

Submit your article to this journal $₫$

Q View related articles $\sqsubset$ 


\section{XXXIX.-Descriptions of Chalcidites discovered in Valdivics by C. Darwing, Esq. By Fraxcis Watker, F.L.S.}

Pachylarthrus Sariaster, Mas. I"iridis, antcunc lutere, podes flati, ales limpidsc.-Fem. Cupreus, antomec nigre, podes fulti, fomora virillin.

Mas. Corpus crassum, convexum, nitens, viride cuprea-varium, scite sonamenm, parce pubescens : caput magnum, transtersum, breve, viride, thorace Jatius; vertex latus, cupreo-varius; frous impressa, abrupte declivis : oculi rufi, mediocres, non extantes : os flatum; palpi maxillares clavati; anicmie luter, graciles, subfiliformes, 13-articulatic, thorace panllo longiores; articulus

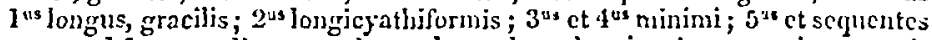
usque ad $10^{\text {um }}$ mediocres, subxquales; clava longiconica, acuminata, articulo $10^{\circ}$ fere duplo longior: thorax oritus, robustus : prothornx transversus, brevis, antice angustus: mesothoracis scutum longitudine latius; parapsidun sutura bene determinatx, postice approximatx; paraptera et epimera nagna; scutellum subconicum, sat magnum : metathorax uncdiocris, obconicus, declivis : petiolus sat longus: abdomen rhombiforme, cuntractum, lave, glabrum, latitudine non longius, thoracis dimidii longitudine; segmentum $1^{\text {wa }}$ maximum, dorsum obternens: pedes flari, simplices, subarquanles; coxæ virides; ungues et pulvilli fusci : alie limpida ; squanula luter; nervi fulvi; nerrus humeralis ulnari multo longior, radialis ulnari vix brevior, cubitili duplo longior; stigma minutum.

Fem. Cupreus, parum nitens : caput thorace vix latius: palpi maxillares simplices: antemne nigra, subfiliformes, thorace non longiores; articulus 2 as cyathiformis; $5^{\text {us }} \mathrm{ct}$ sequentes usque ad $10^{\text {um }}$ subiequales, approximati; clava conica, articulo $10^{\circ}$ longior : petiolus brevis : abdomen oratum, supra fere planum, subtus carinatum, apice acuminatum et attenuatum, thorace vix Jongius; segmentum $1^{\text {uar }}$ magmom, "2un et sequentia brevia : pedes fulvi; coxe virides; femora viridia, apice fulva. (Corp. long. lin. $1-1 \frac{1}{3}$; alar. lin. 1 s $-2 \div$.)

Dicyclus Lynastes, Fem. Viridi-ancus, antenne nigra, pedes fulci, fonora cuea, ale limpillc.

Corpus breve, convexum, viridi-xneum, nitens, scitissine squamcum, parce hirtum : caput transversum, breve, thorace paullo latius; vertex latus; frons impressa, abrupte declivis: oculi ruf, mediocres, non extantes: antennx nigra, graciles, subclarate, thorace vix longiores; articulus $2^{\text {us }}$ lon. gus; $2^{\text {"s }}$ cyathiformis; $3^{\text {us }} \mathrm{et}$ 4 $^{\text {us }}$ minimi ; $5^{\text {us }} \mathrm{ct}$ sequentes usque ad $10^{\text {urn }}$ subaequales, approximati; clava conica, compressa, acuminata, arriculo $10^{\circ}$ multo longior: thorax ovatus : prothorax eransrerstrs, brevis, antice angustus: mesothoracis scutum longitudine latius, parapsidum suture remotre, sat bene determinate, postice approximate; paraptera et epimera magna; scutcllum subconicum, mediocre: metathorax brevi obconicus, declivis : pctiolus brevis: abdomen subrotundum, supra fere planum, subtus carinatum, apice acuminatum, thorace brevius; scgment:um 1 val magnum : pedes folvi, simplices, subrequales; coxx mnex; femorn xnea, apice fulva; tarsi apice fusci: alic limpida ; squanule picen; ncrvi fulvi; nerrus humeralis ulnari multo longior, radjalis uluari vix brevior, cubitali longior; stigma minutum. (Corp. long. lin. $\frac{3}{7}$; alar. lin. I $\frac{1}{3}$.)

\section{Lamprotatus Natta, Mas. Firidi-cyaneus, antenne nigra, pedes flavi,} ala limpida.

Corpus angustum, sublineare, convexum, viridi-cyancum, nitens, scitissime squameum, parce hirtum: caput transversum, breve, siride, thorace latius; vertex latus, anco-varius; frons impressa, abrupte declivis : oculi rufi, 


\section{Mr. Walker's descriptions of Chalcidites from Valdivia.}

mediocres, non cxtantes : antcmur nigre, graciles, subfiliformes, thorace vix breviores; articulus l"s longus, sublinearis; $2^{\text {us }}$ longicyathifor mis, $33^{\text {"o }}$ ct $4^{\text {us }}$ minimi; $5^{\text {"1s }}$ ct sequentes usque ad $10^{\text {um }}$ subequales, nproximati; clava longiovata, acuminata, articulo $10^{\circ}$ multo Iongior : thorax Iongi-subovatus: prothora: transversns, mediccris, antice paullo angustion: necouthoracis scutwin longitudine latius; parapsidum sulure bane deterininataz, postice aljproximata; paraptera ct epimera magnat; scutellum stibcouicun, medincre: metathorax magnus, declivis, obconicus : petiolus sat lougus: abdomen bre viosatun, leve, glabrum, thorace multo brevius : pedes flavi, simplices, subicguales; cosa virides; tarsi apice fusci : ala limpida; squnnu':a fusca ;

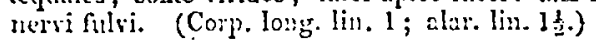

Lamprotatus Bisaltes, Mas. Fineo-riridis, antenne niyre, pedes favi, femora viridia, alce limpida.

Corpus convexum, aneo-viride, nitens, scitissine squameum, parce lirtum: caput transtersum, breve, thoracis latitudine; vertex latus; frons impressa, abrupte declivis : oculi rufi, mediocres, non extantes: antemue nigra?: thorax ovatus: prothorax transwersus, brevissimus: mesothoracis scutum longitndine latius; prapsidum suturx bene determinate, postice approximatx; paraptera ct cpimcra magna; scutcllum conicum, mediocre: metahorax brevi-obconicus, declivis : petiolus brevissimus: abdomen sublinearc, leve, glabrum, ferc planum, thorace angustius et multo brevins; scomentum I un maximum : pedes flavi, simplices, sabrequalcs; coxac viridcs; femora viridia, apice thava; tarsi apice fusci : ala limpida; squamula picce; nervi fusci ; uervus lumeralis uluari fere duplo longior, radialis ulnari paullo brevior, cubitali paullo longior; stigma parrum. (Corp. long. liu. 1 ; alas. lin. 1:.)

Isamprotatus Orobia, Fem. Firidis, cupreo-rarius, antenna nigra, pedes $r u f$, femora basi viridia, ala limpidee.

Viridis, convexus, nitens, scitissime squamcus, parce pubescens : caput transversum, mediocre, thoracis latitudine; vertex s:t latus; frons abrupte declivis, ad antennarum receptionem excarata: oculi picci, mediveres, non extantes: antcmuce subfiliformes, ni'rre, graciles, pubescentes, thoiace non longiores; articulus I" longus, gracilis ; 2" conspicui; $5^{\text {"s }}$ longicyathiformis; $\sigma^{\text {us }}$ et sequentes lireviores : thorax longiovatus: prothorex mediocris, transversus, antice angulum utringue finge'ns non angustior: mesothorasis scutun longitudine vix latius; parapsidun sutura bene determinatix, postice approximatre; paraptera et cpincra magna; scufellum mediocre, conicum : metathorax mediocris, declivis, obconicus: petiolus brevis : abdomen oratum, nitens, live, ferc glabrun, apice acuminatum, thorace paullo brevius: sermenta antica megna, postica breviora: oriductus mon exertus : pedes graciles, recti, subxquales, pallide rufi, pubesccutcs : coxw rirides; trochanteres picca; fomora basi viridia; tarsi apice fusci : alæ limpirla, mediocres; squamula viridi-piecæ; nervifusci; ncrvus lumeralis ulnari fere duplo longior, cubitalis radiali brevior; stigmat minutum. (Corp. long. lin. 1, alar. lin. 2t.)

Far. 6.-Capitis vertex viridi-ancus : thoracis disctus cupreo-aneus : abdomen cupreo-rarim.

I'tcromalus Mcgarcus, Fem. Iriridi-aneus, antenne nigra, pedes flavi, femora viridia, tibia fusco-rinctce, alce limpida.

Corpus viridi-xucun, convexum, parum nitens, scitissine squameum, parce jubsescens : cajut transrersun, bresc, thonace jinullo latius; vertex sat latus; frons abrupte declivis, cxcavata : oculli picci, mediocres, non extantes: antemne nigra', subcliwatic, pubescentes, sat graciles, thorace non longiores; 


\section{Mr. Walker's descriptions of Chalcidites from Valdivia. 273}

articulus $1^{\text {ns }}$ viridis : thorax breviovatus : prothorax brevissimus, supra vix conspicuus : mesothoracis scutum latum ; parapsidum suture non bene determinatie; scutellum subrotundum, mediocre: metathorax brevis, declivis, obconicus: petiolus brevissimus: abdomen longiovatum, nitens, lreve, fere glabrum, supra planum, subtus carinatum, apice acuminatum, thorace longius et angustius : pedes flavi; coxa virides; femora viridia, apice flava; tibia fusco-cincte; tarsi apice fusci : ala limpida; squamulæ picea; nervi fulvi; nervus humeralis ulnari multo longior, cubitalis radiali multo brevior;

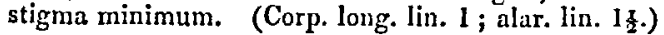

Closterocerus Xenodice, Mas et Fem. Viridis cyareo-varius, abdomen cupreum, antenna nigra, pedes flavi, femora viridia, tibia fusco-cincta, ala fusco-maculate.

MIas. Corpus sublineare, angustum; depressum, læete viride, nitens, læve, parce hirtum : caput transversum, brevissimum, inter oculos impressum; rertex sat latus; frons abrupte declivis: oculi mediocres: antenna subsetacex, nigra, hirta, thorace non longiores: thorax ovatus: prothorax brevissimus: mesothoracis scutum longitudine vix latius; parapsidum sutura vix conspicux; scutellum subovatum : metathorax obconicus, declivis, mediccris : petiolus brevissimus : abdomen sublineare, cupreum, basi cyaneoviride, thorace angustius non longius: pedes flavi; coxa virides; femora viridia; tibia fusco-cincta ; tarsi apice fusci : als mediocres, ciliata, fulvotincta, in discum obsolete fusco-maculate; squamula piceæ; nervi fulvi; nervus ulnaris humerali multo longior, radialis vix ullus, cubitalis brevissimus in ala discum abrupte declivis; stigma minimum.

Var. $\beta$.-Tibia omnino fuscae : alis macula vix conspicua.

Var. \%.-Ala omnino limpida.

Fem. Cyaneo-viridis: antenna setacea, thorace breviores: abdomen ovatum, thorace paullo brevius vix angustius. (Corp. long. lin. $\frac{1}{3}-\frac{2}{3}$; alar. lin. $\frac{1}{2}-1$.)

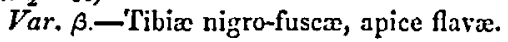

Platygaster Paches, Mas et Fem. Atra, antenna nigra, pedes nigri, tarsi picei, ala subfusca.

Mas. Corpus convexum, atrum, nitens, lave, fere glabrum : caput transversum, breve, thorace vix latius; vertex sat latus; frons abrupte declivis : oculi picci, parvi, non extantes : antentu nigre: thorax ovatus : prothorax brerissimus, supra vix conspicuus : mesothoracis scutun longitudine latius; parapsidum suture non bene deterininate; scutellum subrotundum, non productum : metathorax parvus, obconicus, declivis : petiolus brevis : abdomen longiovatum, thorace paullo angustius non longius; segmentum $1^{\text {um }}$ magnum, $2^{u m}$ et sequentia breviora: pedes nigri, subaquales; femora clavata; genua picea; tibire clavata; tarsi picei: ala subfisca; squamulx picex. (Corp. long. lin. $\frac{1}{2}$; alar. lin. $\frac{3}{4}$.)

Fem. Caput thoracis latitudine: antenna subclavata: abdomen longioratum, apice acuminatum, thorace angustius et paullo longius.

Inostemma Quinda, Fem. Atra, antennce frua, apice fusca, pedes favi, alce limpides.

Corpus atrum, angustum, sublineare, convexum, læve, nitens, pubescens: caput transversum, subrotundum, thorace vix latius; vertex latus; frons abrupte declivis : oculi parvi, non extantes : antenna subclavata, flava, apice fusca : thorax ovatus: prothorax brevissimus, supra vix conspicuus: mesothoracis scutum transiersum, sulcis 2 indistinctis postice approximatis; parapsidum suturx non bene determinatæ; scutellum obconicum, non productum : metathorax brevi-obconicus, declivis : petiolus crassus, brevissimus: abdomen longiovatum, glabrum, apice acuminatum, thorace paullo longius

Ann. \& Mag. N.Hist. Tol.x. 


\section{Mr. W. Thompson on the Crustacea of Ireland.}

non angustius; segmentum jum maximum : pedes favi, simplices, subrquales: alæ limpidx, mediocres; squamulæ picca; nervi fulvi; nervus subcostalis ala basi emissus nervulum in discum rejiciens et spatio brevi ante costa medium in stioma subfurcatum decedens : nervus quoque spurius alie basi-emissus in discum excurrens et nerro subcostali nervuloque triangulum fingens. (Corp. long. lin. $\frac{1}{2}$; alar. lin. 1.)

Romilius (11. g. *) Zotale, Fem. Ater, antenne nigra basi fulva, pelles fulci, alc limpida.

Corpus longum, angustum, sublineare, atrum, vix convexum, obscurum, pilis canis pubescens, subtilissime et confertissime punctatum : caput transversum, breve, subrotundum, thorace vix latius; vertex latus; frons conrexa, non impressa: oculi parvi, non extantes: antennæe nigrax, graciles, subclavatx, prope os insertx, thorace non breviores; articulus $1^{\text {a }}$ longus,

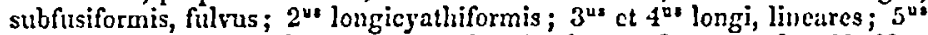
et sequentes ad $10^{\text {um }}$ breves, approximati, clavam fingentes longifusiformem : thorax longiovatus : prothorax brevissimus, postice excavatus : mesothoracis scutum magnum, trisulcatum, longitudine vix latius; sulci laterales postice approximati; scutellum parvum, semicirculum fingens : metathorax mediocris, obconicus : petiolus brevissimus : abdomen longifusiforme, subtus convexum, apice acuminatum, thorace multo longius; $\operatorname{scgmentum~} 1^{\mathrm{nta}}$ breve; $2^{u_{m a}}$ et $3^{\mathrm{um}}$ magria; $4^{\mathrm{nm}}$ et $5^{\mathrm{um}}$ paullo breviora; $6^{\mathrm{m}}$ adhuc brevius : pedes fulvi, longi, gracilcs, simplices, subxquales; tarsis articulus $1^{\text {us }}$ longus, $2^{\text {us }}$ multo brevior, $3^{\text {us }}$ adhue hrevior, $4^{\text {us }} 3^{\circ}$ brevior, $5^{\text {us }} 4^{\circ}$ paullo longior : alac mediocres, limpida, abdominis apicem dum quietem agunt non attingentes; squamula fulva; nerri fulvi; nervus subcostalis aln basi cmissus ad costa medium cam attingens et fere ad ala apicem percurrens; nervus 2" quoque ala basi emissus in discum excurrens ibique furcatus, furca antica nervo subcostali triangulum fingens, furca postica ad alæ marginem posticum decedens; nervus cubitalis rectus, sat longus, nerro subcostali ubi costam attingit emissus, stigmate terminatus minuto. (Corp. long. lin. 13; ; alar. lin. 23.)

[To be continued.]

XL.-The Crustacea of Ireland. Order Decapoda. By Wur. Thompson, Esq., Vice-Pres. Nat. Hist. Society of Belfast.

\section{CRUSTACEA. Order Decapoda.}

\section{Decapoda Brachyurt.}

Macropodia phalangium, Lcach, Mal. pl. 23. f. 6; Desmar. p. 155. pl. 23 . f. 3.

Stenorhyncus phalangium, Edw. Crust. tome i. p. 279.

Cancer phalangium, Penn. Brit. Zool. vol. iv. p. 11. pl. 9. f. 3, edition 1812:

This species has already becn recorded by Templeton as " not uncommon on the Irish coast," and by Mr. J. V. T'bompson as "very abundant in the deep water of the harbour of Cove." Ent. Mag. vol. iii. p. 371 .

It has occurred very commonly to my scientific friends and myself when dredging in the loughs of Strangford and Belfast; and to Mr.

- Scelio Duris (Monogr. Chalcid., ii. 61.) also belongs to this gemus. 